\title{
LEVI FLAT HYPERSURFACES WHICH ARE NOT HOLOMORPHICALLY FLAT
}

\author{
ERIC BEDFORD AND PAOLO DE BARTOLOMEIS
}

\begin{abstract}
A real analytic, Levi flat hypersurface $S \subset \mathbf{C}^{n}$ is locally biholomorphically flat. It is shown here that if $S$ is Levi flat and $C^{\infty}$, then in general it is not possible to flatten $S$, even in a local, "one-sided" sense.
\end{abstract}

If $S \subset \mathbf{C}^{n+1}$ is a smooth hypersurface whose Levi form vanishes identically then $S$ is a 1-parameter family of complex manifolds of dimension $n$. If $S$ is real analytic, then locally one may choose holomorphic coordinates $z^{*}$ such that $S=\left\{\operatorname{Re} z_{n+1}^{*}=0\right\}$. Let us fix $p \in S$ and fix one side of $S$ at $p$; we let $D^{+}$denote a small one-sided neighborhood of $S$ at $p$, which, after shrinking, will be again denoted by $D^{+}$.

We consider the possibility of a local one-sided flattening of $S$ in the following sense.

There exists a holomorphic map $F: D^{+} \rightarrow\left\{z \in \mathbf{C}^{n+1}\right.$ :

$\left.\operatorname{Re} z_{n+1}>0\right\}$ such that if $\left\{\zeta_{j}\right\} \subset D^{+}$and $\lim _{j \rightarrow \infty} \operatorname{dist}\left(\zeta_{j}, S\right)$

$=0$ then $\lim _{j \rightarrow \infty} \operatorname{dist}\left(F\left(\zeta_{j}\right),\left\{\operatorname{Re} z_{n+1}=0\right\}\right)=0$.

It is easily seen that $S$ cannot be flattened in the sense of (1) if the Levi form of $S$ does not vanish identically (cf. condition (c) of the lemma). Related results have been obtained by Henkin [4] and Pinčuk [5]. The point of this paper is that Levi flatness is not sufficient for the surface $S$ to be flattened.

The complex $n$-manifolds in $S$ form a $C^{\infty}$ foliation $\mathfrak{F}$ of $S$. A related problem is to ask whether $\mathfrak{F}$ can be extended to a holomorphic foliation of $D^{+}$. (Recall that a foliation of codimension 1 is holomorphic if locally there are coordinates $z^{*}$ such that the leaves are given as $\left\{z_{n+1}^{*}=c\right\}$.)

LEMMA. The following are equivalent near a point $p$ of a smooth Levi flat hypersurface $S \subset \mathbf{C}^{n+1}$.

(a) $S$ can be flattened in the sense of (1),

(b) $S$ can be flattened in the sense of (1), and we may take the holomorphic mapping $F$ in (1) to be smooth on $\bar{D}^{+}$and $F: D^{+} \rightarrow F\left(D^{+}\right)$is to be a biholomorphism,

(c) there is a pluriharmonic function $h \in C^{\infty}\left(\bar{D}^{+}\right)$such that $h>0$ on $D^{+}$and $h=0$ on $S \cap \bar{D}^{+}$, and

(d) there is a $C^{\infty}$ foliation $\tilde{\mathfrak{F}}$ of $D^{+} \cup\left(S \cap \bar{D}^{+}\right)$which is holomorphically trivial on $D^{+}$and such that $\tilde{\mathfrak{F}}=\mathfrak{F}$ on $S \cap \bar{D}^{+}$.

Received by the editors March 6, 1980.

1980 Mathematics Subject Classification. Primary 32F15, 32H99.

(C) 1981 American Mathematical Society 0002-9939/81/0000-0166/\$02.00 
Proof. (a) $\Rightarrow$ (b) If $F$ is the mapping given by (1), then $\operatorname{Re} F_{n+1}$ is pluriharmonic on $D^{+}$and $\lim _{\zeta \in D^{+}, \zeta \rightarrow s} \operatorname{Re} F_{n+1}=0$. Thus

$$
\operatorname{Re} F_{n+1} \in C^{\infty}\left(D^{+} \cup\left(S \cap \bar{D}^{+}\right)\right) \text {. }
$$

It follows, then, that $F_{n+1}$ is also $C^{\infty}$. Now we may assume that $p=0$, and that $\partial / \partial z_{j}$ is tangent to $S$ at 0 for $1<j \leqslant n$. Thus we may replace the original mapping $F$ by $\left(z_{1}, \ldots, z_{n}, F_{n+1}(z)\right)$. By the Hopf lemma $\partial F_{n+1} / \partial z_{n+1} \neq 0$, so this is a local biholomorphism.

(b) $\Rightarrow$ (c) This holds because we may take $h=\operatorname{Re} F_{n+1}$.

(c) $\Rightarrow$ (d) If we let $\tilde{h}$ denote the pluriharmonic conjugate of $h$, then $H=h+i \tilde{h}$ $\in \mathcal{O}(D) \cap C^{\infty}\left(D^{+} \cup\left(S \cap \bar{D}^{+}\right)\right)$. We define the leaves of $\tilde{\mathfrak{F}}$ as the level sets $\left\{z \in \bar{D}^{+}: H(z)=c\right\}$. By the Hopf lemma, $\partial h \neq 0$ on $S$, so the leaves are smooth, and $\tilde{\mathfrak{F}}$ is holomorphic on $D^{+}$. Since the leaves of $\mathfrak{F}$ are uniquely determined by integrating $\partial h$ on $S$, it follows that $\tilde{\mathfrak{F}}$ extends $\mathfrak{F}$.

(d) $\Rightarrow$ (a) If $d \operatorname{Im} z_{n+1}$ is the normal covector to $S$ at $p=0$, then we let $\Gamma^{+}=\left\{z \in D^{+}: z_{1}=\cdots=z_{n}=0\right\}$. Shrinking $D^{+}$suitably, we may find a conformal equivalence $f: \Gamma^{+} \rightarrow\{\zeta \in \mathbf{C}: \operatorname{Re} \zeta>0\}$ such that $f(0)=0$ and $\operatorname{Re} f=$ 0 on $\bar{\Gamma}^{+} \cap S$.

We may also assume that each leaf $M$ of $\tilde{F}$ is closed and intersects $\Gamma^{+} U$ $\left(S \cap \bar{\Gamma}^{+}\right)$in exactly one point. Now we define $\tilde{f}$ on $D^{+} \cup\left(S \cap \bar{D}^{+}\right)$by

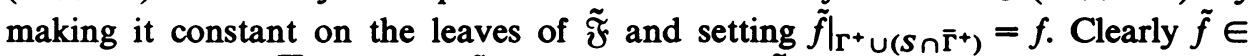
$C^{\infty}\left(D^{+} \cup\left(S \cap \bar{D}^{+}\right)\right)$, and $\tilde{f} \in \mathcal{O}\left(D^{+}\right)$since $\tilde{\mathfrak{F}}$ is holomorphic. Now $F=$ $\left(z_{1}, \ldots, z_{n}, \tilde{f}\right)$ gives the desired map in (a).

C. Rea [6] has given an example of a $C^{\infty}$ surface $S$ such that the foliation $\mathfrak{F}$ does not have an extension to be holomorphic in a two-sided neighborhood of $p$.

Let us assume that $\operatorname{Im} d z_{n+1}$ is normal to $S$ at $p=0$ and that $\operatorname{Im} d z_{n+1}$ points toward $D^{+}$. We may parametrize the foliation $\mathfrak{F}$ of $S$ by $G: \Delta^{n} \times I \rightarrow C^{n+1}$, $I=[-1,1] \subset \mathbf{R}, \Delta=\{\zeta \in \mathbf{C}:|\zeta|<1\}$.

$$
G(z, t)=(z, \varphi(t)+g(z, t))
$$

$\varphi(t) \in C^{\infty}(I), \varphi^{\prime} \neq 0, \varphi^{\prime}(0)>0, g(z, t) \in C^{\infty}\left(\Delta^{n} \times I\right), g(0, t)=0$, and $g$ is holomorphic in $z$ for fixed $t$.

Locally, then, we have $S=G\left(\Delta^{n} \times I\right)$. It will also be useful to parametrize $S$ slightly differently.

Let $\gamma=\varphi(]-\varepsilon, \varepsilon[)$ be the image of $]-\varepsilon, \varepsilon[$ under $\varphi$, and let $\Gamma$ be a small neighborhood of $\gamma$ in $\mathbf{C}$ such that $\Gamma=\Gamma^{+} \cup \gamma \cup \Gamma^{-}$, where $\Gamma^{ \pm}$are connected open sets. We may also write $S=G\left(\Delta^{n} \times \gamma\right)$ where we set

$$
G(z, \eta)=\left(z, \eta+g\left(z, \varphi^{-1}(\eta)\right)\right)
$$

for $z \in \Delta^{n}, \eta \in \gamma$. Since the graph of $g\left(z, \eta_{0}\right)$ for a fixed $\eta_{0} \in \gamma$ is the (unique) leaf of $\mathfrak{F}$ passing through $\left(0, \eta_{0}\right)$ we see that $S$ uniquely determines $g(z, \eta)$ in (3).

For functions $\varphi, a \in C^{\infty}(]-\varepsilon, \varepsilon[)$, we will use the following extendibility criterion.

There is an analytic function $A(\eta) \in \mathcal{\theta}\left(\Gamma^{+}\right) \cap C^{\infty}\left(\Gamma^{+} \cup \gamma\right)$ such that $A(\eta)=a\left(\varphi^{-1}(\eta)\right)$ for all $\eta \in \gamma$. 
REMARK. We note that (4) is a nontrivial criterion which is not satisfied for general $\varphi, a \in C^{\infty}(]-\varepsilon, \varepsilon[)$. For example, we may take $\varphi(t)=t$ and let $a^{+}, a^{-} \in$ $C^{\infty}(\mathbf{R})$ be functions that are nowhere real analytic but such that $a^{ \pm}(t)$ extends to be holomorphic in $\{\operatorname{Im} \zeta \gtrless 0\}$. Then the pair $\left(\varphi, a^{+}+a^{-}\right)$does not satisfy (4) on any interval.

THEOREM. Let the surface $S$ be a $C^{\infty}$, Levi flat hypersurface in $\mathbf{C}^{n+1}$, and let us represent $S$ locally in the form (3). Then $S$ can be locally flattened at $0 \in S$ in the sense of (1) if and only if the pair $(\varphi(t), g(z, t))$ satisfies (4) holomorphically in $z$, i.e. there is a function $\tilde{g} \in \mathcal{O}\left(\Delta^{n} \times \Gamma^{+}\right) \cap C^{\infty}\left(\Delta^{n} \times\left(\Gamma^{+} \cup \gamma\right)\right)$ such that $\left.\tilde{g}\right|_{\Delta^{n} \times \gamma}=$ $g\left(z, \varphi^{-1}(\eta)\right)$.

Before giving the proof we state a corollary.

COROLlary. There is a $C^{\infty}$, Levi flat hypersurface $S$ that cannot be mapped (in the local one-sided sense of (1)) to a real analytic Levi flat surface $S^{\prime}$ in such a way that $\lim _{\zeta \rightarrow p, \zeta \in D^{+}} F(\zeta)=p^{\prime} \in S^{\prime}$ exists.

The corollary follows from the Theorem since we may biholomorphically flatten $S^{\prime}$ in a neighborhood of $p^{\prime}$ and we may choose $S$ to be given by a pair $(\varphi, g)$ which does not satisfy (4).

This is analogous to a result of Faran [2] for strongly pseudoconvex domains. Faran's result uses the local invariants of Chern and Moser [1] and the boundary regularity of $F$, proved by Fefferman [3]. In our case, there are neither local invariants nor boundary regularity for biholomorphisms.

Proof OF THE TheOReM. First we show that $S$ can be flattened if $\tilde{g}(z, \eta)$ is given. We note that $d g(0, t) / d t \neq 0$, and thus $\tilde{F}: \Delta^{n} \times \Gamma^{+} \rightarrow D^{+}$given by $\tilde{F}(z, \eta)=$ $(z, \eta+\tilde{g}(z, \eta))$ is a biholomorphism of a one-sided neighborhood of 0 . By the Riemann mapping theorem there is a holomorphic equivalence

$$
\psi: \Gamma^{+} \rightarrow\{\zeta \in \Delta: \operatorname{Im} \zeta>0\}
$$

such that $\psi(0)=0$. Restricting to a small neighborhood of 0 , we see that $\psi$ maps $\gamma$ to the real axis. If $\Psi(z, \eta)=(z, \psi(\eta))$ then $\Psi\left(\tilde{F}^{-1}\right)=F$ is the desired mapping for (1).

Conversely, we suppose that $S$ can be flattened and show that $(\varphi(t), g(z, t))$ satisfy (4).

By the Lemma, there is a pluriharmonic $h \in C^{\infty}\left(D^{+} \cup\left(S \cap \bar{D}^{+}\right)\right)$such that $h=0$ on $S$ and $h>0$ on $D^{+}$. Let $\tilde{h}$ be a pluriharmonic conjugate so that $H=h+i \tilde{h} \in \mathcal{O}\left(D^{+}\right)$. We define $\tilde{g}(z, \eta)$ on $\Delta^{n} \times \Gamma^{+}$by

$$
H(z, \eta+\tilde{g}(z, \eta))=H(0, \eta)
$$

for all $z \in\left(\Delta^{\prime}\right)^{n}$ where $\Delta^{\prime} \subset \subset \Delta$ is some smaller disk. Since $\partial H / \partial \eta \neq 0$ on $\Delta \times\{0\}$, we see that we may use (5) to define $\tilde{g}: \Delta^{n} \times \Gamma^{+} \rightarrow \mathbf{C}$ implicitly (recall that we are free to shrink $\left.\Gamma^{+}\right)$. Since $H(0, \eta)$ is analytic in $\eta$ for $\eta \in \Gamma^{+}$we see that $\tilde{g} \in \mathcal{O}\left(\Delta^{n} \times \Gamma^{+}\right) \cap C^{\infty}\left(\Delta^{n} \times\left(\Gamma^{+} \cup \gamma\right)\right)$. Further, since for fixed $\eta_{0} \in \gamma$ the graph of $\tilde{g}\left(z, \eta_{0}\right)$ is the leaf of $\mathfrak{F}$ passing through $\left(0, \eta_{0}\right)$, it follows that $\tilde{g}\left(z, \eta_{0}\right)=$ $g\left(z, \varphi^{-1}\left(\eta_{0}\right)\right)$ and thus $(\varphi, g)$ satisfies $(4)$. 


\section{REFERENCES}

1. S. S. Chern and J. Moser, Real hypersurfaces in complex manifolds, Acta Math. 133 (1974), 213-271.

2. J. Faran, Non-analytic hypersurfaces in C", Math. Ann. 226 (1977), 121-123.

3. C. Fefferman, The Bergman kernel and biholomorphic mappings of pseudoconvex domains, Invent. Math. 26 (1974), 1-65.

4. G. M. Henkin, An analytic polyhedron is not holomorphically equivalent to a strictly pseudoconvex domain, Soviet Math. Dokl. 14 (1973), 858-862.

5. S. I. Pinčuk, Biholomorphic inequivalence of bounded domains with smooth and piecewise-smooth boundary, Dokl. Akad. Nauk SSSR 247 (1979), 554-557. (Russian)

6. C. Rea, Levi-flat submanifolds and holomorphic extensions of foliations, Ann. Scuola Norm. Sup. Pisa Sci. Fis. Mat. 26 (1972), 665-681.

Department of Mathematics, Princeton University, Princeton, New Jersey 08544 (Current address of Eric Bedford)

School of Mathematics, Institute for Advanced Study, Prnnceton, New Jersey 08540

Current address (Paolo de Bartolomeis): Istituto Matematico “U. Dini”, viale Morgagni 67/A, I-50134 Firenze, Italy 